\title{
EUS-guided gastroenterostomy for the management of gastric outlet obstruction: A systematic review and meta-analysis
}

\author{
Umair Iqbal ${ }^{1}$, Harshit S. Khara², Yirui Hu3, Vikas Kumar², Kashif Tufail², Bradley Confer², David L. Diehl² \\ ${ }^{1}$ Department of Internal Medicine, Geisinger Commonwealth School of Medicine, ${ }^{2}$ Department of Gastroenterology and \\ Hepatology, Geisinger Medical Center, ${ }^{3}$ Geisinger Medical Center, Biomedical and Translational Informatics Institute, \\ Danville, PA, USA
}

\begin{abstract}
Gastric outlet obstruction (GOO) is characterized by epigastric pain and postprandial vomiting secondary to mechanical obstruction. Management of GOO is usually focused on alleviating the symptoms of obstruction and can be achieved by surgical gastrojejunostomy or enteral stenting. Recent studies have shown success with EUS-guided gastroenterostomy (EUS-GE) in the management of GOO but data is limited. We, therefore, conducted a meta-analysis to evaluate the safety and efficacy of EUS-GE in the management of GOO. A comprehensive literature review was conducted by searching the Embase and PubMed databases from inception to January 2019 to identify all studies that evaluate the efficacy and safety of EUS-GE in GOO. Our primary outcome was to evaluate technical success and clinical success. Secondary outcomes were to evaluate the need for reintervention and adverse events of the procedure. Twelve studies including 285 patients were included in the meta-analysis. Technical success was achieved in 266 patients with a pooled technical success of $92 \%$ (95\% confidence interval [CI]: $88 \%-95 \%$ ). Clinical success was achieved in $90 \%$ of the patients (95\% CI: $85 \%-94 \%)$. Recurrence of symptoms or unplanned reintervention was needed in $9 \%$ of the patients (95\% CI: $6 \%-13 \%)$ and adverse events were reported in $12 \%$ of the patients $(95 \%$ CI: $8 \%-16 \%)$. The heterogeneity tests among studies were nonsignificant with ${ }^{2}=0$. EUS-GE is a safe and efficacious treatment modality for the management of benign and malignant GOO. Larger prospective studies are needed to further evaluate its utility in GOO.
\end{abstract}

Key words: EUS, enteral stenting, gastric outlet obstruction, gastroenterostomy, gastrojejunostomy

\section{INTRODUCTION}

Gastric outlet obstruction (GOO) is a potential complication of pancreatic or distal gastric malignancy and may present in as many as $15 \%-20 \%$ of patients with pancreatic adenocarcinoma. ${ }^{[1,2]}$ Ampullary cancer,

\begin{tabular}{|l|l|}
\hline \multicolumn{2}{|c|}{ Access this article online } \\
\hline Quick Response Code: & Website: \\
\hline & www.eusjournal.com \\
\cline { 2 - 2 } & DOI: \\
\hline
\end{tabular}

lymphoma, and biliary malignancy may also cause GOO. There are also several benign etiologies of GOO including chronic pancreatitis, peptic ulcer

\begin{abstract}
This is an open access journal, and articles are distributed under the terms of the Creative Commons Attribution-NonCommercial-ShareAlike 4.0 License, which allows others to remix, tweak, and build upon the work non-commercially, as long as appropriate credit is given and the new creations are licensed under the identical terms.
\end{abstract}

For reprints contact: reprints@medknow.com

How to cite this article: Iqbal U, Khara HS, Hu Y, Kumar V, Tufail K, Confer B, et al. EUS-guided gastroenterostomy for the management of gastric outlet obstruction: A systematic review and meta-analysis. Endosc Ultrasound 2020;9:16-23.

\section{Address for correspondence}

Dr. Umair Iqbal, Department of Internal Medicine, Geisinger Commonwealth School of Medicine, 100 North Academy Avenue, Danville, PA 17822, USA. E-mail: uiqbal@geisinger.edu

Received: 2019-05-15; Accepted: 2019-11-11; Published online: 2019-12-30 
disease, caustic ingestion, and others. ${ }^{[3-6]}$ Patients usually present with epigastric pain, postprandial vomiting and unable to tolerate an oral diet. Therefore, the primary goal in the management of GOO is to relieve the obstruction so that patients can tolerate an oral diet. ${ }^{[2]}$ This has been conventionally achieved by surgical gastrojejunostomy (SGJ) or enteral stenting (ES). Studies have reported comparable efficacy of both approaches though better short-term outcomes with ES compared to SGJ..$^{[7-10]}$ SGJ can be associated with high morbidity and mortality. ${ }^{[1]}$

EUS-guided gastroenterostomy (EUS-GE) has recently been successfully utilized in the management of GOO ${ }^{[12-24]}$ In EUS-GE, an EUS-guided bypass is made by inserting a lumen apposing metal stent (LAMS) from the gastric lumen to the small bowel distal to the obstruction. Currently, limited evidence exists regarding the efficacy and safety of this procedure, and the literature is mostly limited to small case series and observational studies. We, therefore, conducted a systematic review and meta-analysis of the available literature to evaluate the safety and efficacy of this procedure in benign and malignant GOO.

\section{MATERIALS AND METHODS}

\section{Search strategy}

A systematic literature search was conducted in the PubMed and Embase databases to identify all studies from inception to January 2019 that assessed the efficacy of EUS-GE in GOO. The systematic literature review was independently conducted by two investigators using search terms as follows: (( ("Gastric Outlet Obstruction” [Mesh] OR "Gastric Outlet Obstruction" [tiab])) AND ("Endosonography" [Mesh] OR "endosonography" [tiab])) AND ("Gastroenterostomy" [Mesh] OR "Gastroenterostomy" [tiab]) in PubMed. We also searched Embase using terms, "endoscopic ultrasonography"/exp OR "endoscopic ultrasonography" OR "endoscopic ultrasonography":Ti, ab AND "gastroenterostomy"/exp OR “gastroenterostomy” OR "gastroenterostomy”:Ti, ab and "gastrojejunostomy"/exp OR "gastrojejunostomy" OR "gastrojejunostomy":Ti, ab AND, "endoscopic ultrasonography"/exp OR "endoscopic ultrasonography" OR “endoscopic ultrasonography”:Ti, ab.

\section{Selection criteria}

Eligible studies were observational studies or case series that evaluated the efficacy of EUS-GE for the management of GOO. Included studies should also report technical success and safety outcomes of the procedure. We did not include individual case reports. A sample size of the study did not restrict its inclusion in the meta-analysis. There was no language restriction for the study to be included in the meta-analysis. Abstracts that are not fully published were also eligible for inclusion if they reported our outcomes of interest. The Newcastle-Ottawa quality assessment scale was used to evaluate the quality of observational studies in three areas: the recruitment of cases and controls, the comparability of the two groups and the outcome of interest of the cohort study. We also evaluated the quality of the case series using NIH quality assessment tools. The results of the methodological quality assessment did not influence the eligibility of the studies. This study was conducted in agreement with the Preferred Reporting Items for Systematic Reviews and Meta-Analysis statement. ${ }^{[25]}$

\section{Data extraction}

A structured data collection form was used to extract the following data from each study: demographic information, indications for EUS-GE, etiology of GOO, technical success, clinical success, adverse events, recurrence of symptoms or need for reintervention, procedure time if reported, and procedural technique utilized. Technical success was defined as successful EUS-guided deployment of the LAMS. Clinical success was defined as the ability to tolerate oral intake after the procedure. The data regarding stent diameter was inconsistently reported therefore was not abstracted. To ensure accuracy, data extraction was independently performed by two investigators and was reviewed by the third investigator. Any disagreement was resolved by mutual consensus.

\section{Statistical analysis}

Meta-analyses were conducted for each included outcome using the random-effects model. Incidence rates and 95\% confidence intervals (CIs) were estimated for binary outcomes from included studies. Each included study's pooled estimates and measures of variability were used to generate Forrest plots. Publication bias was evaluated by Egger's test. Variability between included studies was assessed via heterogeneity tests using the $I^{2}$ statistics. The $I^{2}$ statistic was calculated to quantify the proportion of between-study heterogeneity attributable to variability in the association rather than sampling variation. A value of $I^{2}$ of $0 \%-25 \%$ represented no significant 
heterogeneity, 26\%-50\% represented low heterogeneity, $51 \%-75 \%$ represented moderate heterogeneity, and $I^{2}>75 \%$ represented high heterogeneity. All analyses were conducted in RStudio (Version 1.0.136, RStudio, Inc. Boston, MA, USA) using the "Meta" and "Metafor" package. ${ }^{[26]}$

\section{RESULTS}

The initial search revealed 274 articles, 23 in PubMed and 251 in Embase. After the removal of duplicates, review articles, and editorials, 22 articles underwent title and abstract review and 17 articles were selected for full manuscript review. Articles were excluded if they evaluated the efficacy of EUS-GE for any indication other than GOO. If the article was presented in abstract form and fully published, we only included the fully published article to avoid duplication. After a full review, 12 articles including 285 patients were included in the final meta-analysis. ${ }^{[12-15,17-24]}$ We believe that Chen et al. 2017 study comparing EUS-GE with ES and Khashab et al. 2017 study comparing EUS-GE and SGJ has a similar patient population in EUS-GE group. ${ }^{[16,17]}$ Therefore, we excluded Chen et al. 2017 study from the meta-analysis to avoid duplication. ${ }^{[16]}$ Figure 1 elaborates on the systematic literature review process of our study.

Among 12 included studies 3 were case series, 1 prospective study, and 8 retrospective studies. 7 of

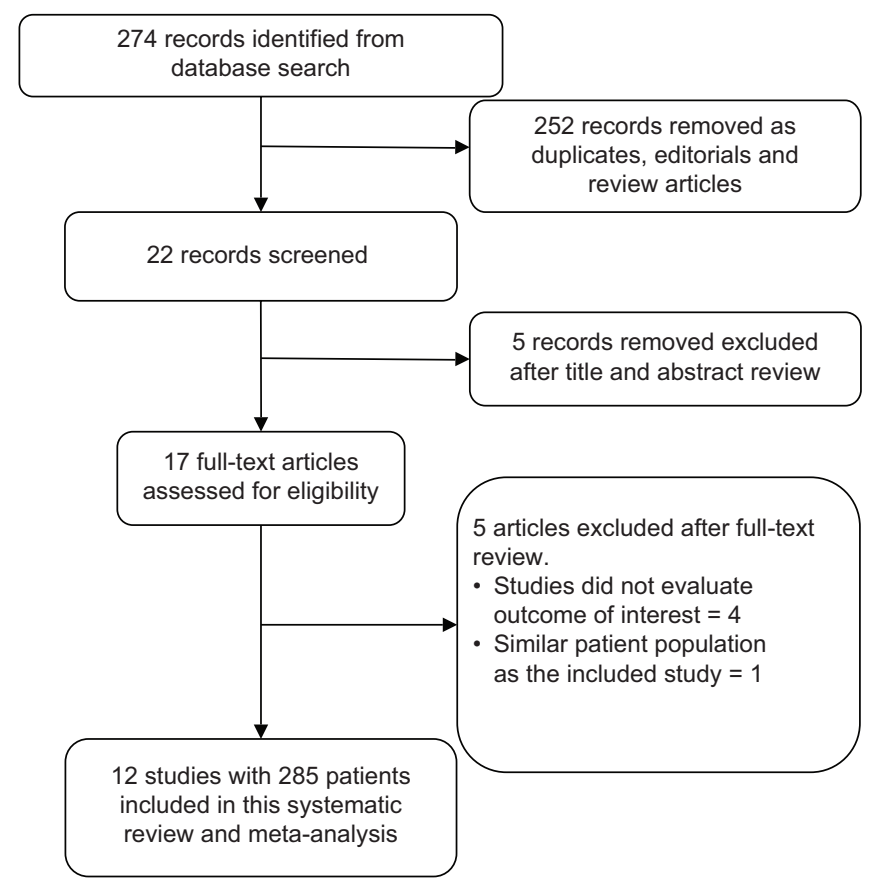

Figure 1. Literature review process the included studies were good quality studies while 5 were of fair quality. EUS-GE was performed in 202 patients with GOO secondary to malignancy while 83 patients had benign etiologies of GOO. EUS-GE was performed with different procedural techniques with direct EUS-GE is the most common approach, followed by balloon-assisted technique and EUS-guided double-balloon occluded gastrojejunostomy bypass (EPASS). Baseline characteristics and quality assessment of the included studies were reported in Table 1.

Technical success was achieved in 266 of 285 patients who underwent EUS-GE with a pooled technical success of 92\% (95\% CI: 88\%-95\%) [Figure 2]. Clinical success was achieved in 249 patients with a pooled clinical success rate of $90 \%$ (95\% CI: $85 \%-94 \%)$ [Figure 3]. The technical and clinical success of the individual study was reported in Table 1. Adverse events occurred in 28 patients with a pooled incidence of 12\% (95\% CI: 9\%-17\%) [Figure 4]. Adverse events are reported in detail in Table 1. Recurrence of GOO symptoms and the need for unplanned re-intervention occurred in 16 patients with a pooled incidence of 9\% (95\% CI: 6\%-13\%) [Figure 5]. There was no statistical heterogeneity existed among the included studies $\left(I^{2}=0 \%, P>0.05\right)$. Egger's test indicated no publication bias as the symmetry existed in the funnel plot $(P>0.05)$ [Figure 6].

\section{DISCUSSION}

This is the first meta-analysis done to evaluate the safety and efficacy of EUS-GE in the management of GOO. GOO carries a poor prognosis and is associated with high morbidity and mortality. GOO increases the risk of malnutrition and significantly impacts the quality of life. Therefore, the primary goal in these patients is to relieve the symptoms of obstruction that would support them tolerate the oral intake. ${ }^{[2]}$ SGJ has been conventionally done for the palliation of symptoms but it is invasive and associated with significant morbidity. ${ }^{[11]}$ Endoscopic stenting of the duodenum is an alternative to surgical bypass and has comparable efficacy and has the advantage of being less invasive and shorter time to tolerate oral intake..$^{[7-11,27,28]}$ However, as compared to surgical bypass, endoscopic ES has a higher rate of stent occlusion which may require the need for reintervention. ${ }^{[8,11]}$

EUS-GE provides a safe and efficient approach by utilizing LAMS to create a bypass to relieve symptoms 
Iqbal, et al.: EUS-GE for GOO

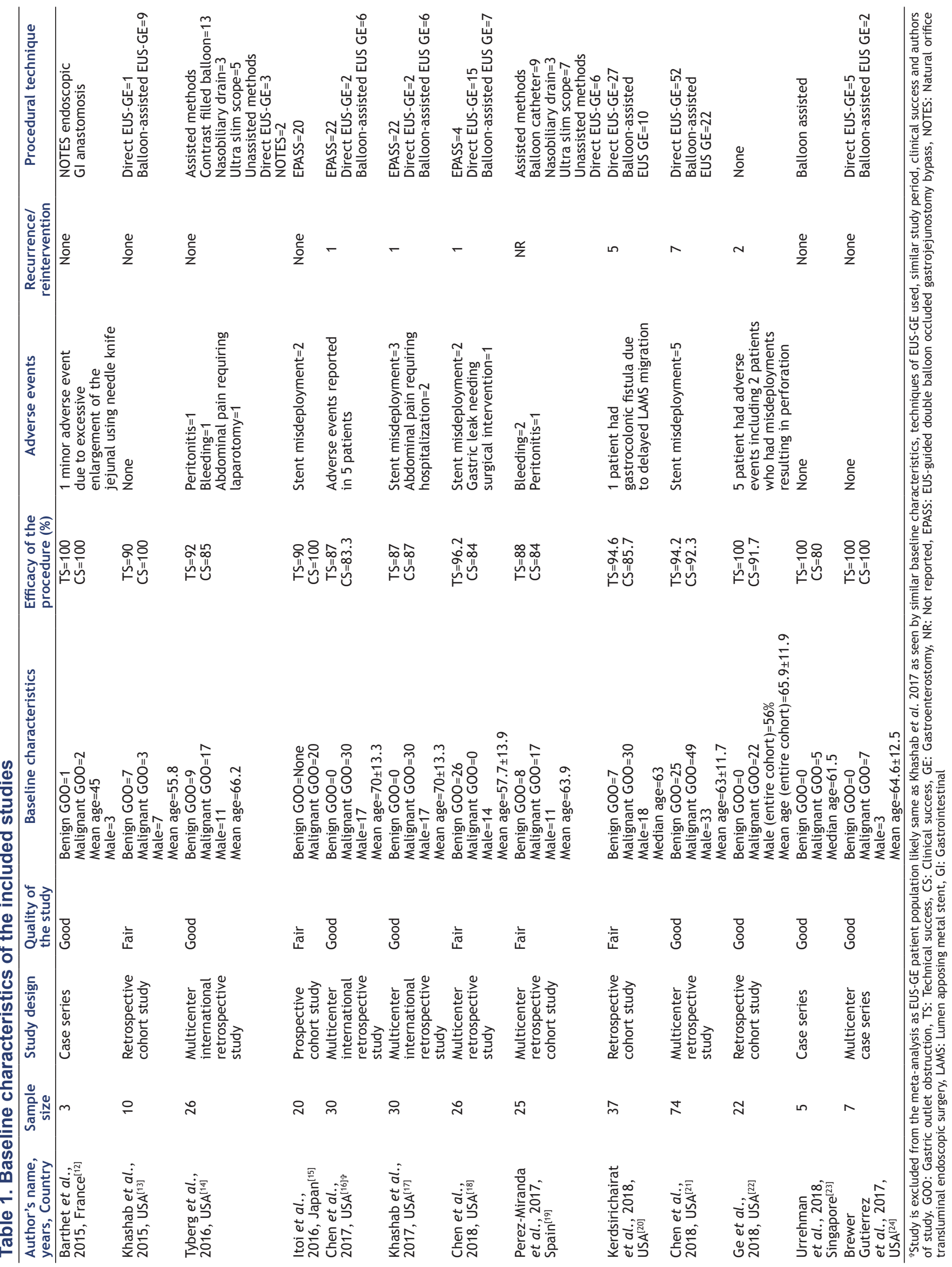




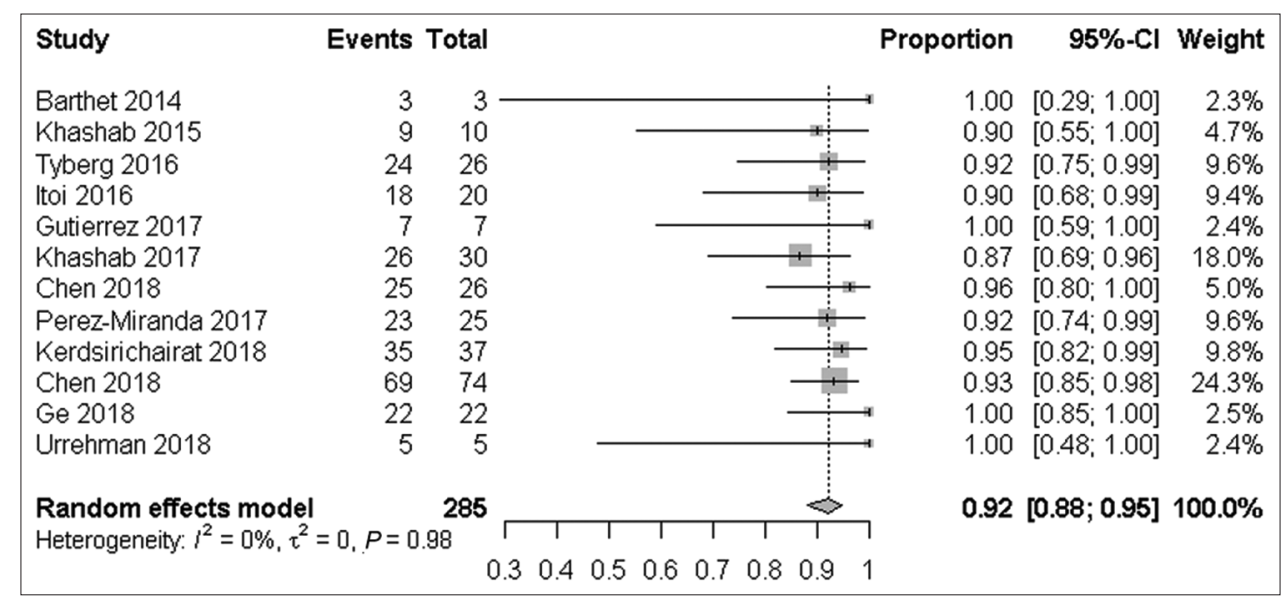

Figure 2. Forrest plot of all studies for technical success

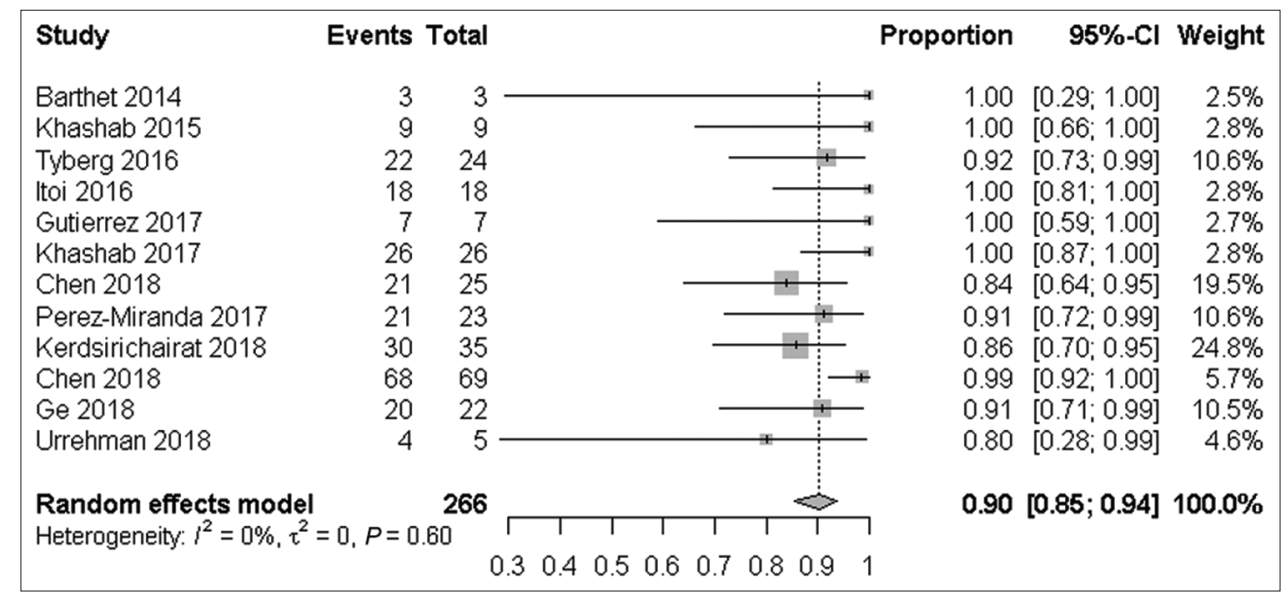

Figure 3. Forrest plot of all studies for clinical success

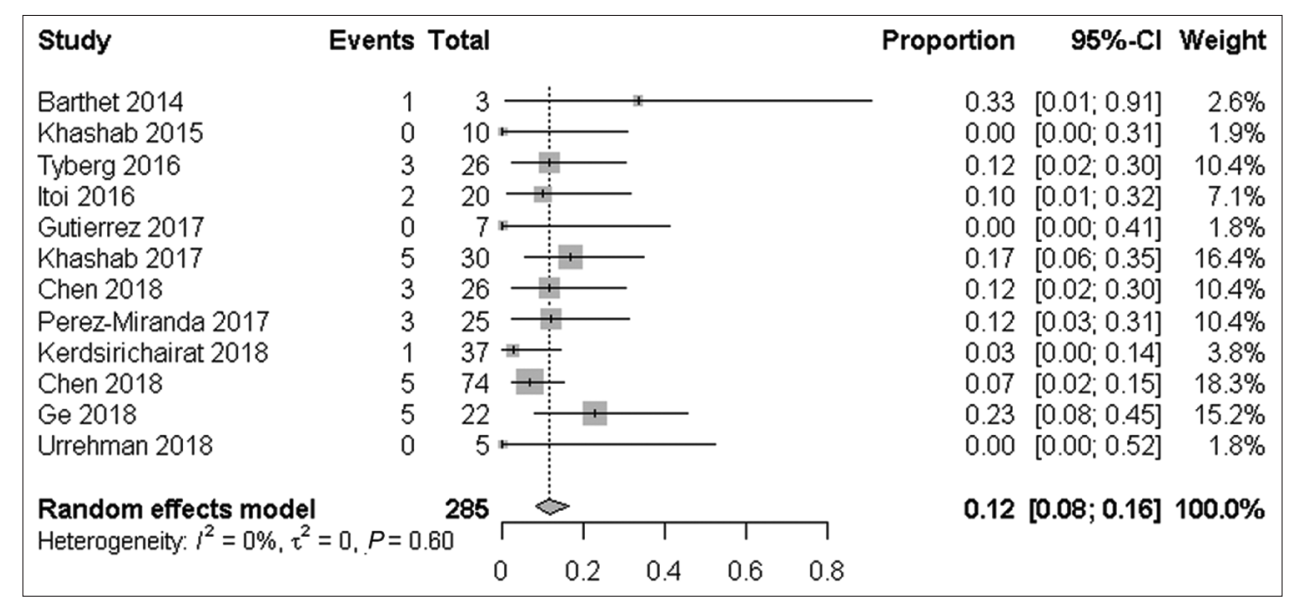

Figure 4. Forrest plot of all studies for adverse events

of obstruction. This meta-analysis revealed technical success and clinical success of EUS-GE ( $92 \%$ and $90 \%$ respectively) which is comparable to that of ES (technical success 96\%-97\% and clinical success $85 \%-90 \%$ ) and SGJ (technical success of $99 \%-100 \%$ and clinical success of $80 \%-90 \%$ ) as demonstrated in previous studies. ${ }^{[7-11]}$ However, there are only a few studies to date that directly compares the efficacy and safety of EUS-GE with SGJ or ES. In a multicenter retrospective study that included 82 patients Chen et al. compared endoscopic duodenal stenting with EUS-GE in patients with malignant GOO. ${ }^{[16]}$ The study found 


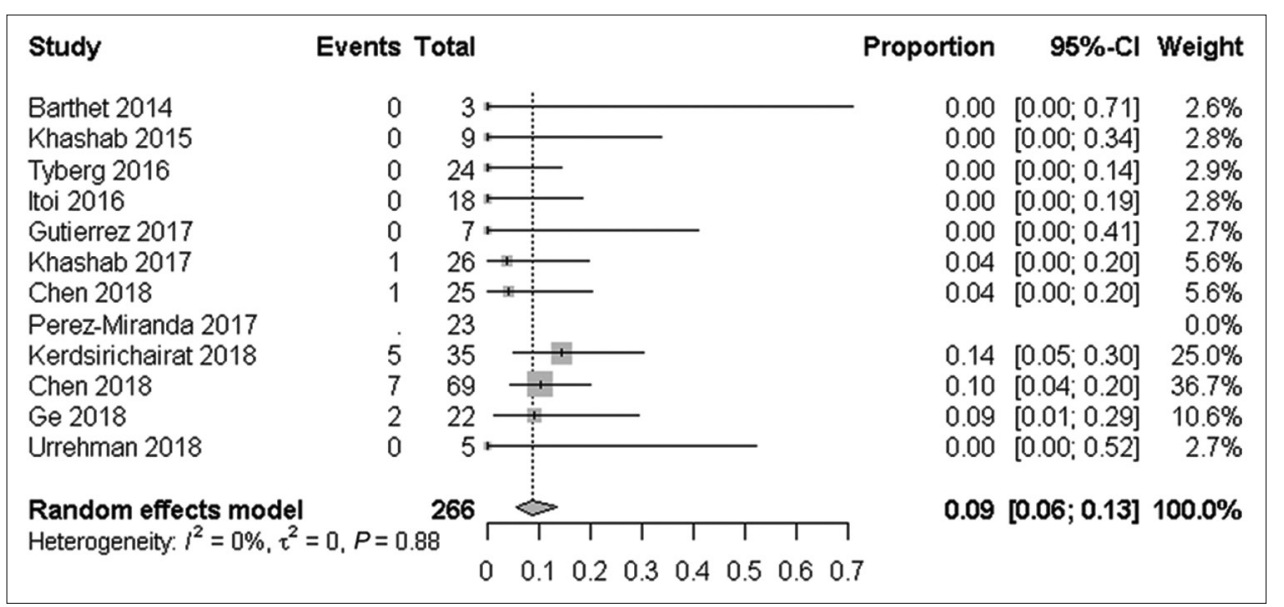

Figure 5. Forrest plot of all studies for recurrence of symptoms or need for re-intervention

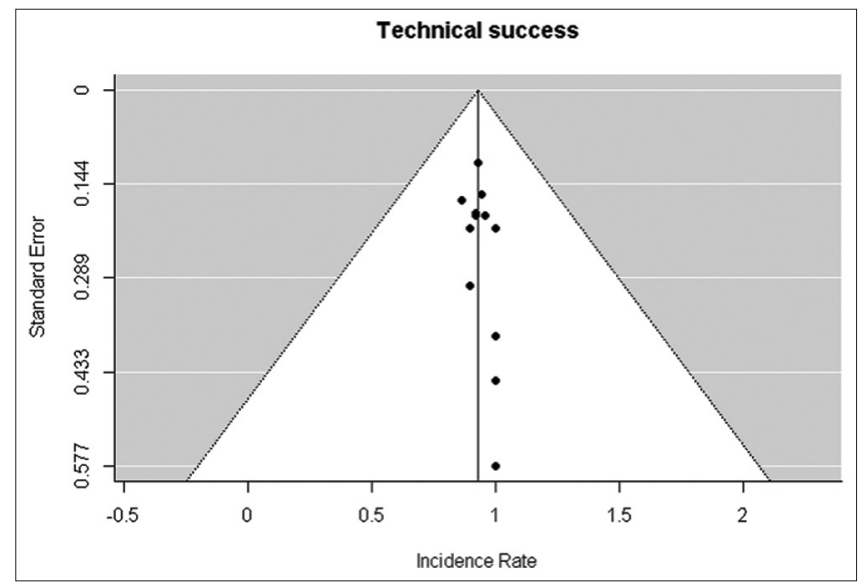

Figure 6. Funnel plot diagram to evaluate publication bias

no significant difference in technical success, clinical success, and rate of adverse events between the two groups. Recurrence of symptoms and the need for repeat intervention was higher in the ES group compare to EUS-GE $(28.6 \%$ vs. $4 \% ; P=0.015) .{ }^{[16]}$ Similarly, in an international multicenter retrospective study by Perez-Miranda et al., there was no difference in the technical and clinical success of EUS-GE and SGJ. ${ }^{[19]}$ However, the rate of adverse events was significantly lower in the EUS-GE group compare to SGJ. Khashab et al. compared EUS-GE with SGJ and showed higher technical success with SGJ. Despite this, there was no significant difference in clinical success, rate of adverse events, or need for reintervention. ${ }^{[17]}$ Kerdsirichairat et al. evaluated the long-term outcomes of EUS-GE using LAMS in 37 patients. This study showed promising long-term outcomes of the procedure with $82.3 \%$ and $77.8 \%$ of patients showed favorable outcomes at 6 and 12 months. ${ }^{[2]}$ There was only one adverse event reported in a patient who developed gastro-colonic fistula due to migration of LAMS.
Although malignant GOO is more prevalent, benign etiologies can also cause GOO. Endoscopic balloon dilation (EBD) has usually been considered as the initial treatment modality but its success largely depends upon the etiology of benign disease. EBD along with eradication of Helicobacter pylori is effective in around $80 \%$ of the patient with GOO secondary to PUD. ${ }^{[3,29]}$ EBD has poor efficacy in the management of strictures secondary to chronic pancreatitis and requires multiple sessions in management of injury to caustic substances. ${ }^{[4-6,30-33]}$ ES has also been considered in the management of benign GOO and has shown favorable results but is associated with stent migration. ${ }^{[10,34]}$ Chen et al. in a study done on 26 patients who underwent EUS-GE due to GOO secondary to benign etiologies demonstrated technical success in $96 \%$ and clinical success in $84 \%$. Therefore, EUS-GE might be a promising procedure in patients who have failed EBD or who have a benign cause of GOO.

EUS-GE is also safe and successful in the management of GOO in patients with altered anatomy. ${ }^{[14,19]}$ It has also been utilized in the management of afferent loop syndrome (ALS) and in the reversal of altered anatomy in patients with previous gastric bypass. ${ }^{[35-38]}$ In a case series of 15 patients with altered anatomy EUS-GE was technically and clinically successful in all patients with complications reported in only two patients. ${ }^{[38]}$ In a multicenter retrospective series of 18 patients with ALS EUS-GE was technically successful in 100\% of the patients. ${ }^{[36]}$ Resolution of symptoms occurred in 16 patients while the other two patients reported improvement in their ALS-related symptoms. Adverse events and re-intervention were required in 3 patients. ${ }^{[36]}$

The optimal technique for EUS-GE is still unclear. Direct EUS-GE carries a risk of inadvertent colonic 
access, but the use of methylene blue infusion into the small intestinal lumen followed by utilization of needle puncture and aspiration of infused bluish material may decrease that risk. Another technique is the injection of contrast via the access needle and evaluation under fluoroscopy to confirm small bowel lumen access vs. colonic puncture. Chen et al. compared the efficacy and safety of direct EUS-GE technique with balloon-assisted EUS-GE. ${ }^{[21]}$ No difference was found between the two groups in technical success, clinical success, rate of adverse events, need for repeat intervention, or survival. However, mean procedure time was significantly lower in direct GE group compared to the balloon-assisted group (35.7 $\mathrm{min} v$ s. $89.9 \mathrm{~min})$. ${ }^{[21]}$ EPASS is also an alternative EUS-GE technique that is not currently available in the United States, and most of the included studies that utilized this technique were conducted in Japan. Future prospective studies are needed to further evaluate any difference in outcomes between different EUS-GE techniques.

The systematic literature search of our study is comprehensive, the sample size is fair, and all the included studies are fair to good quality; however, there are some limitations to our meta-analysis. First, the majority of the included studies are case series and retrospective studies which may introduce bias in the study results. There are only limited retrospective studies that directly compared the EUS-GE with ES or SGJ. Furthermore, most of the studies are done by the same group of authors; therefore, despite our best efforts to exclude duplication of patients, there is still a chance of this which also may introduce bias in our study results. The sample size of the studies is small to moderate and larger prospective studies are lacking. Finally, although the statistical heterogeneity is insignificant, methodological heterogeneity might still exist given differences in techniques of the procedure, patient population, and expertise of the clinician.

\section{CONCLUSION}

In summary, EUS-GE is an effective treatment modality for the management of benign and malignant GOO and might be associated with lesser adverse effects compared to other techniques. The rate of reintervention and recurrence of symptoms are also lower with EUS-GE. Further research is needed to directly compare the efficacy of EUS-GE with endoscopic duodenal stenting and SGJ in GOO and to evaluate optimal EUS-GE technique.

\section{Financial support and sponsorship}

Nil.

\section{Conflicts of interest}

There are no conflicts of interest.

\section{REFERENCES}

1. Tendler DA. Malignant gastric outlet obstruction: Bridging another divide. Am J Gastroenterol 2002;97:4-6.

2. van Heek NT, van Geenen RC, Busch OR, et al. Palliative treatment in "peri"-pancreatic carcinoma: Stenting or surgical therapy? Acta Gastroenterol Belg 2002;65:171-5.

3. Solt J, Bajor J, Szabó M, et al. Long-term results of balloon catheter dilation for benign gastric outlet stenosis. Endoscopy 2003;35:490-5.

4. Kochhar R, Dutta U, Sethy PK, et al. Endoscopic balloon dilation in caustic-induced chronic gastric outlet obstruction. Gastrointest Endosc 2009;69:800-5.

5. Chiu YC, Liang CM, Tam W, et al. The effects of endoscopic-guided balloon dilations in esophageal and gastric strictures caused by corrosive injuries. BMC Gastroenterol 2013;13:99.

6. Kochhar R, Sethy PK, Nagi B, et al. Endoscopic balloon dilatation of benign gastric outlet obstruction. J Gastroenterol Hepatol 2004;19:418-22.

7. Nagaraja V, Eslick GD, Cox MR. Endoscopic stenting versus operative gastrojejunostomy for malignant gastric outlet obstruction-a systematic review and meta-analysis of randomized and non-randomized trials. J Gastrointest Oncol 2014;5:92-8.

8. Jeurnink SM, van Eijck CH, Steyerberg EW, et al. Stent versus gastrojejunostomy for the palliation of gastric outlet obstruction: A systematic review. BMC Gastroenterol 2007;7:18.

9. van Halsema EE, Rauws EA, Fockens $P$, et al. Self-expandable metal stents for malignant gastric outlet obstrusction: A pooled analysis of prospective literature. World J Gastroenterol 2015;21:12468-81.

10. Dormann A, Meisner S, Verin N, et al. Self-expanding metal stents for gastroduodenal malignancies: Systematic review of their clinical effectiveness. Endoscopy 2004;36:543-50.

11. Khashab M, Alawad AS, Shin EJ, et al. Enteral stenting versus gastrojejunostomy for palliation of malignant gastric outlet obstruction. Surg Endosc 2013;27:2068-75.

12. Barthet M, Binmoeller KF, Vanbiervliet G, et al. Natural orifice transluminal endoscopic surgery gastroenterostomy with a biflanged lumen-apposing stent: First clinical experience (with videos). Gastrointest Endosc 2015;81:215-8.

13. Khashab MA, Kumbhari V, Grimm IS, et al. EUS-guided gastroenterostomy: The first U.S. clinical experience (with video). Gastrointest Endosc 2015;82:932-8.

14. Tyberg A, Perez-Miranda M, Sanchez-Ocaña R, et al. Endoscopic ultrasound-guided gastrojejunostomy with a lumen-apposing metal stent: A multicenter, international experience. Endosc Int Open 2016;4:E276-81.

15. Itoi T, Ishii K, Ikeuchi N, et al. Prospective evaluation of endoscopic ultrasonography-guided double-balloon-occluded gastrojejunostomy bypass (EPASS) for malignant gastric outlet obstruction. Gut 2016;65:193-5.

16. Chen YI, Itoi T, Baron TH, et al. EUS-guided gastroenterostomy is comparable to enteral stenting with fewer re-interventions in malignant gastric outlet obstruction. Surg Endosc 2017;31:2946-52.

17. Khashab MA, Bukhari M, Baron $\mathrm{TH}$, et al. International multicenter comparative trial of endoscopic ultrasonography-guided gastroenterostomy versus surgical gastrojejunostomy for the treatment of malignant gastric outlet obstruction. Endosc Int Open 2017;5:E275-81.

18. Chen YI, James TW, Agarwal A, et al. EUS-guided gastroenterostomy in management of benign gastric outlet obstruction. Endosc Int Open 2018;6:E363-8.

19. Perez-Miranda M, Tyberg A, Poletto D, et al. EUS-guided gastrojejunostomy versus laparoscopic gastrojejunostomy: An international 
collaborative study. J Clin Gastroenterol 2017;51:896-9.

20. Kerdsirichairat T, Yang J, Brewer Gutierrez OI, et al. Su1362 long-term outcomes of endoscopic ultrasound-guided gastroenterostomy using lumen-apposing metal stents for gastric outlet obstruction: A 4-year cohort. Gastrointest Endosc 2018;87:AB320-1.

21. Chen YI, Kunda R, Storm AC, et al. EUS-guided gastroenterostomy: A multicenter study comparing the direct and balloon-assisted techniques. Gastrointest Endosc 2018;87:1215-21.

22. Ge PS, Young JY, Dong W, et al. 68 EUS-guided gastrojejunostomy with lumen apposing metal stent versus enteral stent placement for palliation of malignant GASTRIC outlet obstruction. Gastrointest Endosc 2018;87:AB46.

23. Urrehman A, JL Khor C, Ka Chin Y, et al. IDDF2018-ABS-0171 Eus-guided gastrojejunostomy using a lumen apposing metal stent in patients with symptomatic gastric outlet obstruction. Gut 2018;67 Suppl 2:A64.

24. Brewer Gutierrez OI, Nieto J, Irani S, et al. Double endoscopic bypass for gastric outlet obstruction and biliary obstruction. Endosc Int Open 2017;5:E893-9.

25. Moher D, Liberati A, Tetzlaff J, et al. Preferred reporting items for systematic reviews and meta-analyses: The PRISMA statement. Ann Intern Med 2009;151:264-9, W64.

26. Higgins JP, Thompson SG, Deeks JJ, et al. Measuring inconsistency in meta-analyses. BMJ 2003;327:557-60.

27. van Hooft JE, Uitdehaag MJ, Bruno MJ, et al. Efficacy and safety of the new WallFlex enteral stent in palliative treatment of malignant gastric outlet obstruction (DUOFLEX study): A prospective multicenter study. Gastrointest Endosc 2009;69:1059-66.

28. Espinel J, Vivas S, Muñoz F, et al. Palliative treatment of malignant obstruction of gastric outlet using an endoscopically placed enteral Wallstent. Dig Dis Sci 2001;46:2322-4.

29. ASGE Standards of Practice Committee, Banerjee S, Cash BD, et al. The role of endoscopy in the management of patients with peptic ulcer disease. Gastrointest Endosc 2010;71:663-8.

30. Aranha GV, Prinz RA, Greenlee HB, et al. Gastric outlet and duodenal obstruction from inflammatory pancreatic disease. Arch Surg 1984;119:833-5.

31. Ciftci AO, Senocak ME, Büyükpamukçu N, et al. Gastric outlet obstruction due to corrosive ingestion: incidence and outcome. Pediatr Surg Int 1999;15:88-91.

32. Rana SS, Bhasin DK, Chandail VS, et al. Endoscopic balloon dilatation without fluoroscopy for treating gastric outlet obstruction because of benign etiologies. Surg Endosc 2011;25:1579-84.

33. Taylor SM, Adams DB, Anderson MC. Duodenal stricture: A complication of chronic fibrocalcific pancreatitis. South Med J 1991;84:338-41.

34. Heo J, Jung MK. Safety and efficacy of a partially covered self-expandable metal stent in benign pyloric obstruction. World J Gastroenterol 2014;20:16721-5.

35. Brewer Gutierrez OI, Irani SS, Ngamruengphong S, et al. Endoscopic ultrasound-guided entero-enterostomy for the treatment of afferent loop syndrome: A multicenter experience. Endoscopy 2018;50:891-5.

36. Dormann AJ, Deppe H, Wigginghaus B. Self-expanding metallic stents for continuous dilatation of benign stenoses in gastrointestinal tract - First results of long-term follow-up in interim stent application in pyloric and colonic obstructions. Z Gastroenterol 2001;39:957-60.

37. Taunk P, Cosgrove N, Loren DE, et al. Endoscopic ultrasound-guided gastroenterostomy using a lumen-apposing self-expanding metal stent for decompression of afferent loop obstruction. Endoscopy 2015;47 Suppl 1:E395-6.

38. Amateau SK, Lim CH, McDonald NM, et al. EUS-Guided endoscopic gastrointestinal anastomosis with lumen-apposing metal stent: Feasibility, safety, and efficacy. Obes Surg 2018;28:1445-51. 